\title{
DESEOS Y PROPOSICIONES
}

\author{
ALBERTO MORETTI \\ Sociedad Argentina \\ de Análisis Filosófico
}

En $A$ Theory of Human Action ${ }^{1}$ A. Goldman ha presentado una teoría especialmente diseñada para comprender la acción humana intencional. En esta nota se considerarán algunos problemas en la caracterización de sus términos básicos y se sugerirá la prescindibilidad de uno de ellos.

Admitida la existencia de propiedades-acto (actos-tipo), Goldman se propone explicar la noción de ejemplificaciones de propiedades; su estrategia consiste en utilizar la noción de acto-caso para definir 'acto-tipo'. Ofrece una definición recursiva de 'acto-caso' que depende del concepto de acto-caso básico y, con esto, "endereza" el camino estratégico, ya que acto-caso básico se define partiendo de la idea de acto-tipo básico. En el capítulo tercero de su libro afirma que

[1] Una propiedad $A$ es un acto-tipo básico para un sujeto $s$ en un tiempo $t$ si y sólo si: si se dieran en $t$ por parte de $s$ el querer ejemplificar $A$ en $t^{\prime}$ y el estar en condiciones estándar respecto de $A$ (carencia de impedimentos $e x$ ternos), entonces aquel querer bastaría (sin mediación de creencias $u$ otros quereres) para causar esa ejemplificación. ${ }^{2}$

Dicho escuetamente: dados el querer hacer $A$ y el porler hacerlo, $A$ es acto-tipo básico si y sólo si el querer hacer $A$ causa 1970.

Goldman, A. A Theory of Humun Action. Princeton: University Press, 2 Se ha modificado de manera inesencial la definición textual (por ejemplo, 'bastaría' resume bastamente la cláusula (b) del original). Supondremos, además, que el recurso a "condiciones estándar" no trivializa la definición. 
inmediatamente el hacer $A$. La noción de querer hacer y, en general, la de querer, surgen, pues, en primer plano.

¿Qué es un querer (want) ? El capítulo cuarto está dedicado a discutir este concepto y a sustentar la tesis de que la relación entre el querer y el actuar es causal. Luego de rechazar la pretensión de que los deseos o quereres sean experiencias sensoriales o actos, Goldman puntualiza las siguientes condiciones necesarias para que un suceso mental $x$ sea considerado un querer: (1) $x$ es un pensamiento (estado de conciencia) favorable hacia algún $y(p .94) ;(2) x$ es un asentimiento a (aceptación de) una proposición optativa (p. 102); (3) $x$ es un suceso mental que puede causar actos (p. 112). Resumiendo, si $x$ es un querer $A$ (de $s$ en $t$ ),

[2] $x$ es el asentimiento (de $s$ en $t$ ) consciente a una proposición optativa acerca de $A$ y $x$ no es acto ni sensación $y$, en ciertas circunstancias, $x$ causaría un acto-caso ( $\mathrm{si} A$ es un hacer básico, para $s$ en $t, x$ causaría un caso de $A$ ).

Cuando analiza el tema del vínculo entre querer y actuar, Goldman parece aceptar

$$
\text { [3] } \underset{x R y))}{\square(x)}(x \text { es querer } \rightarrow(p \rightarrow(\exists y)(y \text { es acto-caso } \wedge
$$

donde $p$ describe las circunstancias en las que el querer tiene "consecuencias típicas", esto es, tiene algún acto como consecuencia, y ' $R$ ' denota la causalidad. De [3] se sigue

[4] $\underset{x R y)) \text {. }}{\square}((\exists x)(x$ es querer $\wedge p) \rightarrow(\exists x)(\exists y)(y$ es acto $\wedge$
$x$

Así, pues, la verdad de [3] (que, en sentido muy lato, establece un vínculo lógico entre querer $\mathrm{y}$ actuar) junto con la afirmación definida por Goldman de que ' $R$ ' denota la causa. lidad $^{3}$

3 La deducción estricta desde [3] del equivalente formal de la cita requiere transformaciones sencillas y una molesta absorción para obtener " $x$ es un querer' en el consecuente antes de concluir con la reposición y distribución 
ensures that if there are any wants and if any of this wants have consequences which are typical of wants, then some wants cause some acts (p. 113).

Por otra parte, de [3] no se sigue

$$
\text { [5] }(x)(x \text { es querer } \rightarrow(\exists y)(y \text { es acto } \wedge \square x R y))
$$

es decir, no se sigue que la relación querer/actuar sea necesaria y por eso puede decirse que the logical relationship [i.e. [3]] ensures a causal relationship [i.e. $R$ ] (p. 112) en contra de Melden, R. Taylor y - se señala- de Abelson y Ch. Taylor, quienes sostendrían algo como [5].

$¿$ Cuál es el fundamento de [3]? The concept of wanting is the concept of something that tends to have certain effects, viz. acts (p. 112). Al parecer, pues, [2] ha de entenderse como el definiens de 'querer'. Esto es, restringiéndonos al querer hacer:

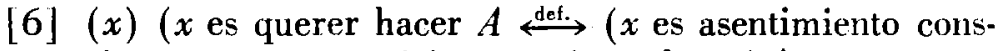
ciente a una proposición optativa sobre $A \wedge x$ no es sensación ni acto $\wedge(p \underset{\text { subj }}{\longrightarrow}(\exists y)(y$ es acto-caso $A \wedge x$ causa $y))))^{4}$

y de este modo se obtiene [3]. Pero en [6] se requiere la noción de acto-caso que depende de la de acto-caso básico, la que, por su parte, se funda en el concepto de acto-tipo básico, en cuya definición, como vimos, es esencial la idea de querer.

de cuantificadores. El esquema de la secuencia es: $p \cdot q \rightarrow r ; p \cdot q \rightarrow p \cdot q \cdot r$; $-(p \cdot q) \vee p \cdot(q \cdot r) ;((-(p \cdot q) \vee p) \cdot(-(p \cdot q) \vee(q \cdot r))) ;-(p \cdot q)$ $\vee(q \cdot r) ; p \cdot q \rightarrow q \cdot r$

Al final se obtiene $\square((\exists x)$ ( $x$ es querer.$p) \rightarrow(\exists x) \quad(x$ es querer . $(\exists y)$ $(y$ es acto $\cdot x R y)))$

${ }^{4} \mathrm{La}$ formulación $p \underset{\text { subj }}{\longrightarrow}(\exists y)$ ( $y$ es acto-caso de A $x$ causa $\left.y\right)$ presenta problemas:

a) puesto que el condicional es subjuntivo, si no se da que $p$, iadmitiremos aún que hay un acto real que $x$ causaría?

b) siguiendo a Quine es posible rechazar la cuantificación sobre los contex- 
Si [6] no se acepta, si, por ejemplo, se suprime el segundo conjuntivo del definiens, aparecen dos problemas: (i) ¿̨Cómo se justifica [3]? y, con ello, ¿en qué se basa la defensa de la relación causal entre querer y actuar? (ii) ¿Cómo afirmar la existencia de quereres inconscientes? En pp. 121/2 se los reconoce como quereres porque, aunque no cumplan la condición de ser sucesos mentales conscientes, presentan la propiedad de causar otros quereres y, por ello, ciertos actos; si esta condición ya no es definitoria, la enmienda que requeriría lo que resta de [6] es muy grande. Es claro que [1] im. plica algo parecido a [3]: de [1] surge que querer un actotipo básico (no todavía un acto en general) es causar un actocaso en ciertas circunstancias. Sobre esta base puede pensarse un fundamento para [3]. Pero [1] es parte principal de la teoría presentada y, entonces, o bien la teoría es acerca del actuar $y$ del querer, en cuyo caso preguntar por el fundamento de [3] es hacerlo por el de la teoría misma, o bien, si querer es primitiva, no es mucho fundamento el que puede darle [1] que la supone.

Si [6] se acepta, ello puede ocurrir en dos formas. Una alternativa es considerar que está en el mismo nivel teórico que [1]; en ese caso la circularidad que se genera obliga a admitir que la teoría no ofrece -como intenta $-{ }^{5}$ una definición explícita de acto sino, tal vez, una definición implícita en relación con la noción de querer. ${ }^{6}$ Otra opción -más apropiada

tos oblicuos que determinarian lo usos del condicional subjuntivo y del predicado de casualidad;

c) 'dado que $p$, se da la causación de un acto-caso $A$ ' no dice que haya determinado caso de $A$ que sea el causado, pero esto es lo que parece decir la fórmula citada.

Estos motivos, entonces, hacen preferible la expresión ' $p \underset{\text { subj }}{\longrightarrow} x$ causa ( $\exists y$ ) $y$ es acto-caso de $A^{\prime}$. Como quiera que sea, estas complicaciones sugieren cambios en todas las fórmulas pertinentes y estas correcciones dejan indemne la dificultad que se quiere señalar en el texto.

5 Observemos de paso que la de Goldman tiende a ser una teoría algo peculiar en cuanto que sus resultados son definiciones explicitas.

6 Goldman asimila la relación entre el concepto de hervor (o de hervido) y su implicación del ser causado por el calor (concedamos este punto de física) con la relación entre acto y ser causado por el querer. Pero 'calor' es definible independientemente de la idea de hervor y la implicación es necesaria cuando se cuenta con una ley física apropiada. 
cuando se advierte que Goldman no ofrece una definición formal de 'querer', a pesar de que dedica un capítulo al tema y de su fuerte preocupación por presentar cuidadosas definiciones de los conceptos de su teoría- consiste en ver en [6] una mera aclaración informal de la noción primitiva de querer. Esto es, la teoría no elucida este concepto y, por tanto, 'acto' en [6] no debe entenderse con la definición derivada de [1] sino en términos preteóricos. Por otra parte, una elucidación del querer que pretenda incorporar a ese concepto la tendencia a causar açtos no podrá usar la noción de acto elaborada por Goldman.

Ahora bien, [6] dice tres cosas acerca del querer: (a) es un estado o suceso mental, pero no es sensación ni es acto, (b) es el asentimiento consciente a una proposición optativa, (c) es algo que tiende a causar actos; y puesto que se trata de una descripción informal, esto que dice ha de considerarse apelando a intuiciones comunes, ya que fuera de una teoría no parece apropiado acudir a conceptos que no se hallen in every. day thought and language i.e. fuera de esa teoría (cf. prefacio, p. vi), a menos que se acompañen de otras elaboraciones teóricas que los apoyen. Con esta perspectiva se observa entonces que (a) pretende surgir de la constatación de que un querer no es una sensación o un sentimiento ni puede ser un acto interno, pero con esto las intuiciones comunes quedan superadas; (b) tampoco proporciona alguna nota positiva para caracterizar el querer (o para abonar su existencia); (c) adelanta un rasgo identificante que, aislado, es insuficiente, ya que ¿por qué no decir simplemente que un querer es una disposición del agente en lugar de serlo de un estado interno suyo? El argumento del conocimiento no observacional ni inferencial de los propios quereres (p. 98) puede atacarse admitiendo que el modo en que un tercero infiere las disposiciones propias no es el modo en que uno las conoce, pero porque no es el modo en que uno las infiere y no porque uno mismo no las infiera (en algún sentido amplio), por ejemplo, a partir de su conducta pasada y las creencias que estén presentes en su mente (un ryleano no tiene que explicar 
el conocimiento de sus quereres más que como el conocimiento de sus disposiciones). El "acceso privilegiado" puede serlo sólo a ciertos recuerdos (acerca de conductas anteriores y de otras creencias) y la "inferencia" puede ser poco más que una veloz reacción mental sintetizada en el lenguaje por las expresiones de deseo. (a) y (c) juntos es poco progreso porque (a) no alcanza a suministrar la entidad que requiere (c) sino que la hace problemática. La condición (b) resulta imprescindible.

Del concepto de proposición optativa no puede decirse que se ha encontrado entre las intuiciones comunes (ni siquiera entre las de los filósofos), de modo que su incorporación reclama algún fundamento teórico. La utilidad de esta noción parece radicar en su contribución al análisis de las inferencias prácticas. Sin embargo, puede sugerirse un análisis que no utiliza este idea novedosa. ${ }^{7}$ En el $\S 2$ del primer capítulo se advierte que el hacer $A$ por parte de $s$ en $t$ será analizado como el ejemplificar la propiedad-acto $A$ por parte de $s$ en $t$, es decir, el ser verdadero $A$ de $s$ en $t$, pero esto no es otra cosa - puede decirse - que el hecho descrito por una oración de la forma $A(s, t) .{ }^{8}$ Si cabe distinguir ahora entre el hecho de que $s$ quiere $A(s, t)$ en $t^{\prime}$ y el objeto mental (de cuestionable existencia) el querer de s en t' que $A(s, t)$, quizá se acepte que el análisis proceda sin referencia a este presunto objeto. Dados: (a) $s$ (en $t$ ) quiere $k$

(b) $s$ (en $t$ ) cree $l$

\footnotetext{
7 Que no la utiliza de manera esencial, como concepto primitivo. Lo que sigue, en caso de aceptarse, puede verse no como la eliminación sino como una reducción del concepto (que lo hace eliminable pero que no lo elimina).

8 Ahora que se roza la cuestión de la "forma lógica" de los entes de que se habla hay lugar para formular algunas preguntas:

1. ¿Por qué no hay actos poliádicos?, ¿cuál es el impedimento filosófico para admitir actos tipo poliádicos? (Cf. p. 14.)

2. ¿Es cierto que igualar (i) el matar a $S$ por parte de $J$, con (ii) el acto de $J$ que causó la muerte de $S$ o el acto mediante el cual $J$ mató a $S$, tiene consecuencias no intuitivas? (Cf. p. 12.) Para Goldman sí porque el matar a $S$ por parte de $J$ no causó el disparo del revólver en tanto que (iii) el apretar el gatillo por parte de $J$ (o el mover su dedo) es la causa del disparo (y ese movimiento digital sería el acto de $J$ que causó la muerte de $S$ en el análisis davidsoniano) y no puede afirmarse que el contexto '... es la causa de $x$ ' sea oblicuo, ni que (iii) sea la causa del disparo bajo la descripción (ii) pero no lo
} 
en lugar de pensar (como Goldman en p. 102) que, a diferencia de la proposición declarativa $l, k$ es una proposición optativa (discutible entidad) acerca de algún acto de $s$, podría adoptarse el siguiente tratamiento: (a') $Q(s, t, A(s, t))$

$$
\text { (b') } C(s, t, p)
$$

donde tanto ' $A(s, t)$ ' como ' $p$ ' se refieren a proposiciones y no a actos-caso $u$ otros sucesos (oblicuidad). Un ejemplo de inferencia práctica, entonces, vendría descrito por

$$
\begin{aligned}
& \text { 1. } Q\left(s, t_{1}, B\left(s, t_{3}\right)\right) \\
& \text { 2. } \mathrm{C}\left(s, t_{1}, A\left(s, t_{2}\right) \rightarrow B\left(s, t_{3}\right)\right) \\
& \text { 3. } Q\left(s, t_{1}, A\left(s, t_{2}\right)\right)
\end{aligned}
$$

y la relación entre los contenidos es, en este ejemplo, el modus ponens (suponemos para simplificar que 1 y 2 son los únicos episodios de creer y querer que intervienen). En general no

sea bajo la descripción (i). Pero ¿por qué no es oblicuo aquel contexto? Su razón es que para afirmar eso debe probarse independientemente la identidad entre (i) y (iii), y este es justamente el asunto en disputa. Pero ocurre que esta identificación es la tesis inicial y no un tema a discutir. $Y$ ¿por qué no hablar de actos bajo ciertas descripciones? Su razón es que esto conduce a afirmar que sólo las acciones bajo descripciones (y entonces dependiendo del lenguaje) causan sucesos. Pero no es así. Esa jerga sólo nos obliga a decir que en las afirmaciones sobre relaciones causales (no en las relaciones causales mismas) es esencial el modo en que se describen los relata, pues hay descripciones insuficientes que no hacen referencia a las propiedades pertinentes aunque se refieran al mismo relatum.

3. 'El ejemplificar por parte de $J$ la propiedad de mover su dedo índice' ino es un modo breve de referirse al ejemplificar por parte de $J$ la propiedad de mover su dedo índice en ciertas circunstancias? (lo que se explicita verbalmente de lo que ocurre no modifica lo que ocurre, y lo que ocurre, desde el punto de vista que Goldman rechaza, es un único suceso). Precisadas las circunstancias ¿no resulta que la propiedad referida en aquella frase es la misma que la referida en 'el ejemplificar por parte de $J$ la propiedad de matar a $S$ ' aunque las dos descripciones tengan sentidos diversos? Que Goldman utiliza a veces este modo de abreviar surge de lo siguiente: correr es una propiedad diferente de correr entre las ocho y las nueve, sin embargo el correr por parte de $s$ es siempre en cierto tiempo $t$, de modo que si $s$ corrió entre las ocho y las nueve ¿realizó dos actos distintos: el correr (de s) entre las ocho y las nueve y el correr entre las ocho y las nueve (de s) entre las ocho y las nueve?

4. En relación a consecuencias no intuitivas: de que se ha producido el ejem. plificar por parte de $s$ la propiedad de mover lentamente el dedo indice se sigue intuitivamente que se ha producido el ejemplificar por parte de $s$ la propiedad de mover lentamente el dedo índice. Si mover el índice $(M)$ y mover el indice lentamente $(M L)$ no se refieren a la misma propiedad, entonces de $M L(s, t)$ no se sigue $M(s, t)$. 
es preciso que esta relación entre contenidos sea deductiva: podría ocurrir que la creencia pertinente fuera "inductiva". De este modo se evita el recurso a proposiciones optativas junto con la necesidad de desarrollar principios lógicos especiales, referidos a tales proposiciones, para juzgar la relación entre los contenidos de las inferencias prácticas. ${ }^{\circ}$ Seguramente para tales juicios se necesitarán algunas leyes naturales que gobiernen la relación $Q$, pero incorporar una relación distinta de la de creencia entre sujetos y proposiciones declarativas es teóricamente (ontológicamente, dirán algunos) menos riesgoso e intuitivamente más confortable que recurrir a una inesperada especie del incómodo género de las proposiciones.

Si aún se deseara hablar de quereres cabría definir:

$x$ es el querer que $A(s, t)$ de $s$ en $t^{\prime}$ def. $x$ es el asentimiento en $t^{\prime}$ de $s a A(s, t) \wedge p \underset{\text { subj }}{\longrightarrow} x$ causa $A(s, t)$

$\mathrm{y}$, mutatis mutandi, se replantearían las posiciones en pugna respecto del vínculo querer/actuar y las definiciones de los conceptos básicos.

* Que sólo hay principios lógicos acerca de proposiciones declarativas, esto es, que también la lógica de la inferencia práctica involucra sólo proposiciones "declarativas", es una opinión que ha sido sostenida, mediante recursos diferentes, por varios autores (Cf. von Wright, G. H. "On So-called Practical Inference" Acta Sociologica, Vol. 15, No. 1; Kenny, A.J.P. "Practical Reasoning and Rational Appetite" en su Will, Freedom and Power, Blackwell, 1975). 